\title{
Impact of Raman Amplification on a 2 Tbit/s Coherent WDM System
}

\author{
Paola Frascella, Student Member, IEEE, Cleitus Antony, Member, IEEE, Simon J. Fabbri, Fatima C. \\ Garcia Gunning, Member, IEEE, Paul Gunning, William McAuliffe, Derek Cassidy, Andrew D. Ellis
}

\begin{abstract}
The impact of hybrid EDFA/Raman amplification on a spectrally efficient coherent-wavelength-divisionmultiplexed (CoWDM) optical communication system is experimentally studied and modeled. Simulations suggested that $23 \mathrm{~dB}$ Raman gain over an unrepeatered span of $124 \mathrm{~km}$ singlemode fiber would allow a decrease of the mean input power of $\sim 6 \mathrm{~dB}$ for a fixed bit error rate (BER). Experimentally we demonstrated 1.2dB $Q$ factor improvement for a $2 \mathrm{Tbit} / \mathrm{s}$ sevenband CoWDM with backward Raman amplification. The system delivered an optical signal-to-noise ratio of $35 \mathrm{~dB}$ at the output of the receiver preamplifier providing a worst case BER of $2 \times 10^{-6}$ over 49 subcarriers at $42.8 \mathrm{Gbaud}$, leaving a system margin (in terms of $\mathrm{Q}$-factor) of $\sim 4 \mathrm{~dB}$ from the forward error correction threshold.
\end{abstract}

\section{Index Terms - Coherent WDM, Raman amplification}

\section{INTRODUCTION}

$\mathrm{R}$ ECENT high capacity and high information spectral density (ISD) systems have used a combination of techniques, such as polarization multiplexing, frequency orthogonality and phase selectivity [1], [2]. In particular, Coherent WDM (CoWDM), which employs phase control to enhance the orthogonality between adjacent frequencies when matched filters are not available, is a promising candidate to achieve high ISD. CoWDM has been demonstrated to be compatible with existing dispersion maps, tolerant to dispersion and nonlinearities using direct and real-time detection, and is well-suited for Ethernet transport [2], [3].

Accompanying this change to higher ISD formats, there is renewed interest in Raman amplification to enable increased reach and capacity of WDM systems [4]. The major advantage of distributed Raman amplification (DRA) is the reduced equivalent noise figure compared to lumped amplification [5][7] for the same nonlinear impact. Whilst Raman amplification has been already used to transmit high-ISD WDM [8], the enhancement in Q-factor has not been quantified yet for

Manuscript received Dec 9, 2010. This material was supported by Science Foundation Ireland under Grants 06/IN/I969 and 07/SRC/I1173.

P. Frascella, C. Antony, S. J. Fabbri, F. C. Garcia Gunning, and A. D. Ellis, are with Photonic Systems Group, Tyndall National Institute, Lee Maltings, Prospect Row, and Department of Physics, University College Cork, Cork, Ireland (e-mail: paola.frascella@tyndall.ie).

P. Gunning is with BT Innovate \& Design, Adastral Park, Martlesham Heath, Ipswich, IP5 3RE, UK

W. McAuliffe, and D. Cassidy are with BT Ireland, Grand Canal Plaza, Dublin 4, Ireland.

Copyright $@ 2011$ IEEE systems with orthogonal and closely-spaced multi-carriers.

In this letter, we demonstrate for the first time the impact of Raman amplification on a CoWDM system over $124 \mathrm{~km}$ of unrepeatered field-installed single-mode fiber (SMF) operated by BT Ireland. We analyze the counter-play of DRA in enhancing and/or decreasing the penalty due to noise and signal distortion from fiber transmission for a $2 \mathrm{Tbit} / \mathrm{s}$ CoWDM, spread over seven independent bands and a total of 49 subcarriers at 42.8 Gbaud. An analytical model is introduced in order to identify high Q-factor operating areas. For such long unrepeatered transmission reaches using legacy installed SMF, the DRA is beneficial, with $1.2 \mathrm{~dB}$ Q-factor enhancement compared to lumped amplification [3].

\section{EXPERIMENTAL SETUP AND EQUIVALENT NOISE FIGURE}

Fig.1 shows the system configuration. The transmitter and receiver were explained in [3]; seven seed lasers spaced at $385.52 \mathrm{GHz}$ passed through two cascaded Mach-Zehnder modulators (MZM), generating seven CoWDM bands each containing seven orthogonal subcarriers spaced at $42.84 \mathrm{GHz}$. After impressing non-return-to-zero on-off keying (NRZOOK) encoding with $2^{31}-1$ PRBS data electrically decorrelated between adjacent subcarriers, the transmitted spectrum had a total power of $8.7 \mathrm{dBm}\left(P_{T X}\right)$ over a $2.8 \mathrm{THz}$ bandwidth and transported a total of 2.1Tbit/s (Fig.2). The transmitted power enabled the inclusion of the dispersion compensating module (DCM)- $9 \mathrm{~dB}$ loss, $-1977 \mathrm{ps} / \mathrm{nm} / \mathrm{km}$ dispersion and $7.6(\mathrm{~W} \cdot \mathrm{km})^{-}$ ${ }^{1}$ nonlinear coefficient- before the first erbium doped fiber amplifier (EDFA \#1 in Fig.1). The unrepeatered $124 \mathrm{~km}$ of installed SMF, looped back from Cork to Clonakilty with a total loss of $26 \mathrm{~dB}$, could be pumped in both forward (FW) and backward (BW) directions with a commercial Raman amplifier including in band and out of band supervisory channels. Compared to post-compensation where the DCM and an additional amplifier are included in the receiver, the optical signal-to-noise ratio (OSNR) benefits of precompensation outweighed the potential penalties associated with the peak-to-average power ratio increase, and so precompensation was used throughout this work. After the transmission fiber, a variable optical attenuator, a power monitor and a $25 \mathrm{~dB}$ small signal gain receiver preamplifier (EDFA \#2) were used, and the OSNR was monitored at the output of the preamplifier with an optical spectrum analyzer. The OSNR measured was defined as the ratio of the signal power in a band $(2.5 \mathrm{~nm})$ over the noise power in $0.1 \mathrm{~nm}$. The 
receiver also comprised two optical filters for subcarrier selection, additional dispersion compensating fiber, and a photodiode for direct detection [3].

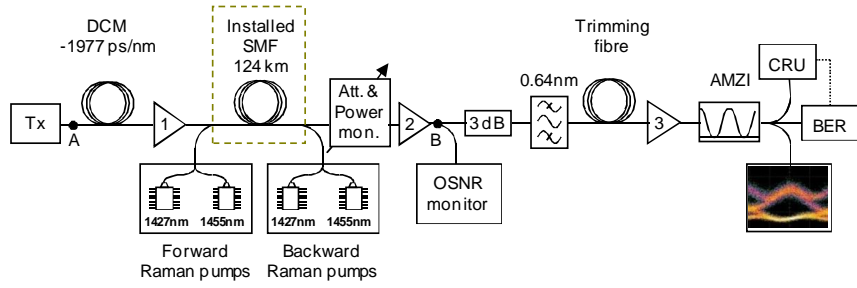

Fig. 1. Experimental setup for hybrid Raman/EDFA transmission over $124 \mathrm{~km}$ installed-SMF of 2Tbit/s OOK CoWDM.

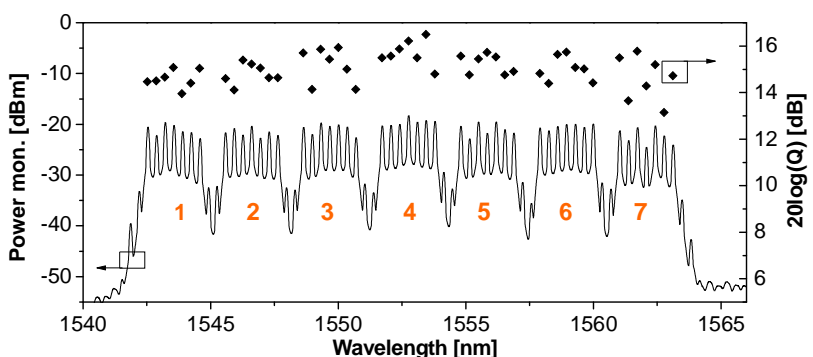

Fig. 2. Spectrum at the transmitter output, with band numeration shown (lefty axis). Calculated Q-factor from BER measurements of all 49 subcarriers with hybrid Raman/EDFA amplification (right y-axis).

We could define an equivalent noise figure $N F_{A B}$ between the points A and B in Fig.1 as:

$N F_{A B}=\frac{N F_{1}}{e^{-\alpha_{D C M} L_{D C M}}}+\frac{N F_{D R A}-1}{e^{-\alpha_{D C M} L_{D C M}} G_{E 1}}+\frac{N F_{2}-1}{e^{-\alpha_{D C M} L_{D C M}} G_{E 1} G_{n e t} 10^{-\alpha_{A T T} / 10}}$

where $N F_{1}$ and $G_{E 1}$ are noise figure and gain of the first EDFA, $N F_{2}$ is the second EDFA's noise figure, $\alpha_{D C M}$ and $L_{D C M}$ are loss coefficient and length of the DCM, $G_{n e t}$ is the net gain over the SMF, and $\alpha_{A T T}$ is the insertion loss in $\mathrm{dB}$ of the variable attenuator and power monitor. $N F_{D R A}$ is the equivalent noise figure of the Raman amplifier which could be expressed as in [5], [9] since BW pumping was the main source of gain in this bidirectional pumping arrangement and [10] in order to take into account of double Rayleigh backscattering (DRB).

Although Raman amplification adds noise (amplified spontaneous emission (ASE) and DRB) [10]) by transforming an optical fiber into a DRA, at a fixed BER it allows lower fiber launch powers and increases the minimum signal power in the fiber. The EDFA following the DRA has also a higher input power, and thus causes a smaller OSNR degradation when compared to the EDFA-only system [3]. Optimization of a DRA system is complex because the distribution of the gain along the transmission fiber reduces the total ASE and improves the OSNR but simultaneously increases the signal distortions that result from Kerr and other nonlinearities. The total nonlinear phase shift is equal to (2):

$\phi_{N L}=\gamma_{D C M} a P_{c h} L_{e f f D C M}+\gamma a P_{c h} e^{-\alpha_{D C M} L_{D C M}} G_{E 1} \int_{0}^{L} G_{n e t}(z) d z$

where $L$ is the SMF length, $\gamma$ and $\gamma_{D C M}$ are the nonlinear coefficients for the SMF and DCM respectively, $P_{c h}=P_{T X} / 49$ is the power per channel or subcarrier, $a$ is an experimentally fitted coefficient that accounts for self-phase (SPM) and crossphase modulation (XPM) between phase matched channels,
$L_{\text {effDCM }}=\left(1-e^{-\alpha_{D C M} L_{D C M}}\right) / \alpha_{D C M}$ represents the effective length for the DCM. $G_{n e t}(z)$ is the net gain and $G_{R A}(z)$ the Raman gain, along the SMF, which are given by (3) and (4) respectively:

$$
\begin{aligned}
G_{n e t}(z) & =a_{A t t} \exp \left[-\alpha_{s} z\right] G_{R A}(z) \\
G_{R A}(z) & =a_{x} \exp \left\{g_{R} \mid \eta_{1} I_{B W}(L) e^{-\alpha_{p} L}\left(e^{\alpha_{p} z}-1\right)+\right. \\
& \left.\left.+\eta_{2} I_{F W}(0)\left(1-e^{-\alpha_{p} z}\right)\right] / \alpha_{p}\right\}
\end{aligned}
$$

where $a_{A t t}$ is the insertion loss of the variable attenuator, $\alpha_{s}$ and $\alpha_{p}$ are the SMF loss at the signal and pump wavelengths respectively, $a_{x}$ takes into account additional insertion losses due to the pump couplers, $g_{R}$ is the Raman gain coefficient $\left(\sim 0.68 \times 10^{-13} \mathrm{~m} / \mathrm{W}\right.$ in $\left.\mathrm{SMF}\right)$, and $\eta_{1}, \eta_{2}$ are efficiencies which scale the pump intensities $I_{B W, F W}\left(=P_{F W, B W} / A_{\text {eff }}\right)$ to take account of the double wavelength system.

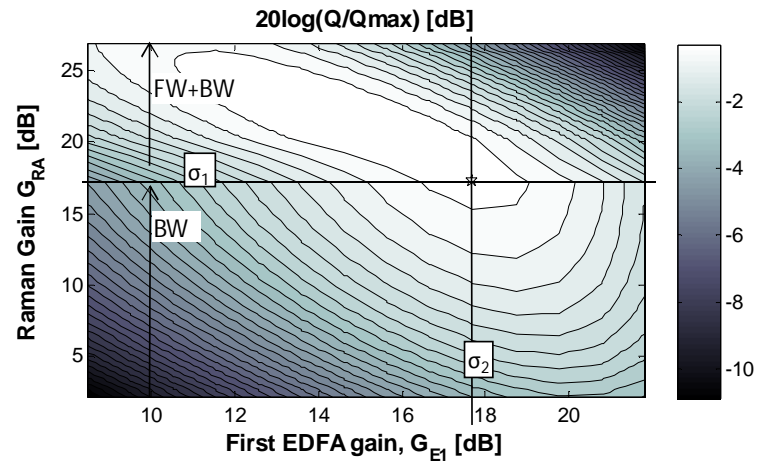

Fig. 3. Normalized Q-factor from simulations as a function of both Raman gain $G_{R A}$ and the first EDFA gain $G_{E 1}$. The spacing of the contour lines is 0.3 .

A Q factor was calculated from the effective signal-to-noise ratio $\left(\mathrm{SNR}_{\mathrm{eff}}\right)$, which sums the linear signal to noise ratio $\left(\mathrm{SNR}_{\text {lin }}\right)$ and nonlinear phase noise (NLPN) [11] as:

$$
\frac{1}{S N R_{\text {eff }}}=\frac{1}{S N R_{\text {lin }}}+\frac{2}{3} \frac{7}{O S N R} \phi_{N L}^{2}
$$

The first term accounts for accumulated ASE and the second is the NLPN variance which depends on the OSNR per channel. In Fig.3 the Q-factor is shown as a function of both the gain in the first EDFA $G_{E 1}$ and the on-off Raman gain $G_{R A}$; an optimum operating area is identified. The Raman gain reached $17.2 \mathrm{~dB}$ when maximum BW pumps only were on.

\section{EXPERIMENTAL RESULTS}

Experimentally two cross-sections $\left(\sigma_{1}\right.$ and $\left.\sigma_{2}\right)$ of Fig.3 were studied. For a fixed Raman gain of $\sim 17.2 \mathrm{~dB}$ (BW only), the gain of the first EDFA was varied in order to evaluate the nonlinear power threshold (Fig.4). The OSNR measurements per band at the transmitter (point A in Fig. 1) were between 38 and $39 \mathrm{~dB}$, and corresponded to the spectrum shown in Fig.2. The flatness over the 49 subcarriers prior to launch into the link was $\sim 5 \mathrm{~dB}$. Fig. 4 shows the Q-factor in $\mathrm{dB}$, calculated directly from the BER measurements for the worst-performing optical subcarrier \#48 (left-axis, squares) at the receiver, against the first EDFA gain $G_{E 1}$ and hence the mean power per subcarrier at the input of the $124 \mathrm{~km}$ transmission fiber (calculated as $1 / 49$ of the total launch power). Similar 
nonlinear performance was observed for a number of randomly selected channels. Fig. 4 also shows the OSNR for the $7^{\text {th }}$ band measured at the output of the first EDFA (\#1 in Fig.1). At low power levels, the Q-factor is degraded by a low OSNR (right-axis, circles); at high powers, the OSNR increases but the Q-factor starts degrading due to nonlinearities. The optimal operating condition was about $1 \mathrm{dBm}$ per subcarrier.

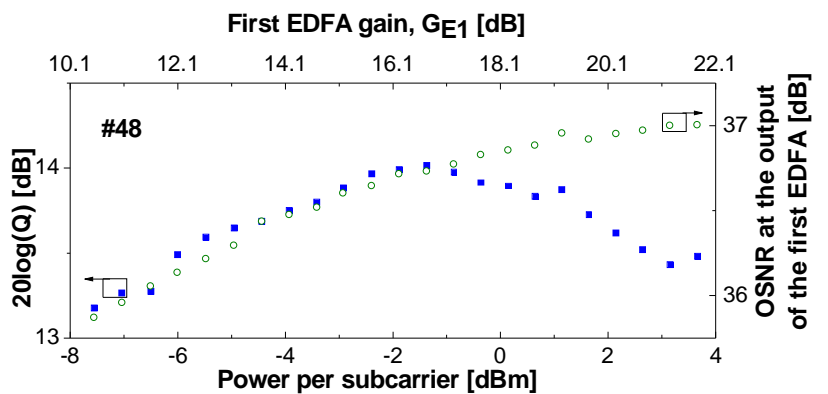

Fig. 4. Calculated Q-factor (left-y axis) from BER measurements for optical subcarrier \#48, and measured OSNR (for the associated band) at the output of the first EDFA (right-y axis) against power per subcarrier at the input of $124 \mathrm{~km}$ installed-SMF (bottom-x axis) and the first EDFA gain (top-x axis).

In order to study the impact of Raman amplification, the Raman gain was varied by changing FW or BW pump powers (reproduction of cross-section $\sigma_{2}$ in Fig. 3). In each case, a dual wavelength pump module was used $(1427 \mathrm{~nm}$ and $1455 \mathrm{~nm}$, $+27 \mathrm{dBm}$ max) and the launch powers of the two pumps were set to be nominally identical, optimizing the gain flatness across the C-band. The Q-factor (calculated from measured BER for optical subcarrier \#48 (left-axis)) against the on-off Raman gain is plotted in Fig.5 as triangles. The measured OSNR is also shown on the right-axis in Fig.5; BW Raman amplification induced a $3 \mathrm{~dB}$ OSNR improvement when compared to an EDFA only amplification system [3], but at maximum BW Raman gain only a $1.2 \mathrm{~dB}$ improvement in the Q-factor is observed. We believe that the saturation in the Qfactor enhancement shown in Fig.3 is due to inter-subcarrier crosstalk. Typically further performance enhancement may be achieved using bi-directional pumping; however in this particular case, the supervisory channel constrained the total loss between the power amplifier and the backward Raman pump, and a degradation of the OSNR was observed if the launch power was reduced by other means. Consequently whilst FW Raman amplification further enhances the gain, Qfactor degradations were observed due to increased nonlinearities at the higher launch power.

The launched power into the installed SMF was fixed at $1 \mathrm{dBm} / \mathrm{ch}$, corresponding to $G_{R A}$ and $G_{E 1}$ of $17.2 \mathrm{~dB}$ (BW Raman only) and $17.7 \mathrm{~dB}$ respectively, indicated by a star in Fig.3. At this operating point, all 49 subcarriers were characterized in terms of BER performance, and the correspondent Q-factors are shown in Fig.2 (right-y axis). An average Q-factor of $15 \mathrm{~dB}$ across the 49 subcarriers was observed after transmission; we believe that the variation of the mean Q-factor of each band is due to the net amplification gain tilt, whilst the large fluctuations observed in bands \#3 and 7 correspond to the flatness deviation of the signal at the transmitter, attributed to the WDM operation of the comb generator. The system $(0.7 \mathrm{bit} / \mathrm{s} / \mathrm{Hz}$ transmitted in a single polarization) had an overall margin in terms of Q-factor of $\sim 4 \mathrm{~dB}$ when related to a threshold BER of $2 \times 10^{-3}$.

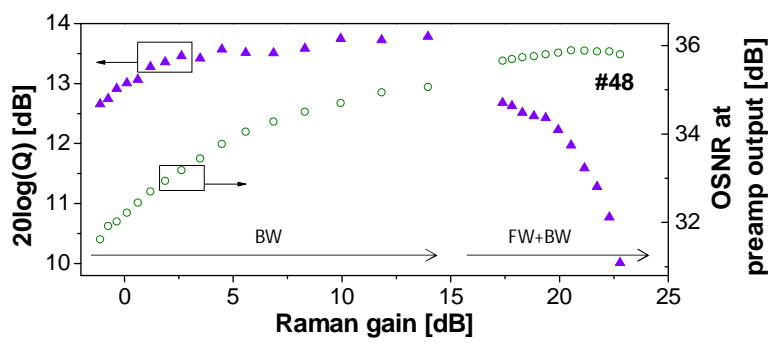

Fig. 5. Calculated Q-factor (left-y axis) from BER measurements for optical subcarrier \#48, and measured OSNR (for the associated band) at the output of the receiver preamplifier (right-y axis) against Raman gain.

\section{CONCLUSION}

We have experimentally demonstrated a $3 \mathrm{~dB}$ improvement in the delivered OSNR for a 2Tbit/s CoWDM system at 42.84Gbaud over $124 \mathrm{~km}$ of field-installed SMF using DRA. The Q-factor improvement was $1.2 \mathrm{~dB}$ compared with EDFA amplification alone.

\section{REFERENCES}

[1] H. Sanjoh, E. Yamada, and Y. Yoshikuni, "Optical orthogonal frequency division multiplexing using frequency/time domain filtering for high spectral efficiency up to $1 \mathrm{bit} / \mathrm{s} / \mathrm{Hz}$ ", Conference on Optical Fiber Communication 2002, ThD1.

[2] A. D. Ellis et al., "Adaptive modulation schemes", Digest of LEOS Summer Topicals 2009, TuD3.2.

[3] P. Frascella, N. Mac Suibhne, F.C.G. Gunning, S.K. Ibrahim, P. Gunning, A.D. Ellis, "Unrepeatered field transmission of $2 \mathrm{Tbit} / \mathrm{s}$ multibanded coherent WDM over 124km of installed SMF", Optics Express vol. 18, no. 24, pp. 24745-24752 (2010).

[4] J. Bromage, "Raman amplification for fiber communications systems", IEEE Journal of Lightwave Technol., vol. 22, no. 1, pp. 79-93 (2004).

[5] E. Desurvire, "Erbium doped fiber amplifiers. Principles and applications", John Wiley \& Sons, New Jersey 2002, pp.121-136.

[6] A. Carena, V. Curri, and P. Poggiolini, "On the optimization of hybrid Raman/Erbium-doped fiber amplifiers”, IEEE Photon. Technol. Lett., vol. 13, no. 11, pp.1170-1172 (2001).

[7] A. Pizzinat, M. Santagiustina, and C. Schivo, "Impact of hybrid EDFADistributed Raman Amplification on a 4 x 40-Gb/s WDM optical communication system", IEEE Photon. Technol. Lett., vol. 15, no. 2, pp.341-343 (2003)

[8] S.L. Jansen, I. Morita, N. Takeda, and H. Tanaka, "20-Gb/s OFDM transmission over 4,160-km SSMF enabled by RF-pilot tone phase noise compensation", in Proc. Opt. Fiber Comm. Conf., Anaheim, CA, Mar. 25-29, 2007, PDP15.

[9] J. Bromage et al., "WDM Transmission Over Multiple Long Spans With Bidirectional Raman Pumping”, IEEE J. Lightwave Technol., vol. 22, no. 1, pp.225-232 (2004).

[10] R.-J. Essiambre, P. Winzer, J. Bromage, and C.H. Kim, "Design of Bidirectionally Pumped Fiber Amplifiers Generating Double Rayleigh Backscattering", IEEE Photon. Technol. Lett., vol. 14, no. 7, pp.914-916 (2002)

[11] J.P. Gordon, L.F. Mollenauer, "Phase noise in photonic communications systems using linear amplifiers", Optics Letters, vol. 15, no. 23, pp.1351-1353 (1990). 